\title{
Chronic Recurrent Multifocal Osteomyelitis (CRMO): Presentation, Pathogenesis, and Treatment
}

\author{
Sigrun R. Hofmann ${ }^{1}$ • Franz Kapplusch ${ }^{1}$ • Hermann J. Girschick ${ }^{2}$ - Henner Morbach ${ }^{3}$. \\ Jessica Pablik $^{4}$ - Polly J. Ferguson ${ }^{5}$ - Christian M. Hedrich ${ }^{1,6,7}$
}

Published online: 27 October 2017

(C) The Author(s) 2017. This article is an open access publication

\begin{abstract}
Purpose of Review Chronic non-bacterial osteomyelitis (CNO) with its most severe form chronic recurrent multifocal osteomyelitis (CRMO) is an autoinflammatory bone disorder. We summarize the clinical presentation, diagnostic approaches, most recent advances in understanding the pathophysiology, and available treatment options and outcomes in $\mathrm{CNO} / \mathrm{CRMO}$.

Recent Findings Though the exact molecular pathophysiology of CNO/CRMO remains somewhat elusive, it appears likely that variable defects in the TLR4/MAPK/inflammasome signaling cascade result in an imbalance between pro- and anti-inflammatory cytokine expressions in monocytes from $\mathrm{CNO} / \mathrm{CRMO}$ patients. In this context, we present previously
\end{abstract}

This article is part of the Topical Collection on Pediatrics

Christian M. Hedrich

Christian.hedrich@liverpool.ac.uk

1 Pediatric Rheumatology and Immunology, Department of Pediatrics, Medizinische Fakultät Carl Gustav Carus, Technische Universität Dresden, Dresden, Germany

2 Vivantes Klinikum Friedrichshain, Children's Hospital, Berlin, Germany

3 Pediatric Rheumatology and Immunology, Children's Hospital, University of Würzburg, Würzburg, Germany

4 Division of Pathology, Medizinische Fakultät Carl Gustav Carus, Technische Universität Dresden, Dresden, Germany

5 Department of Pediatrics, University of Iowa Stead Family Children's Hospital, Iowa City, IA, USA

6 Department of Women's and Children's Health, Institute of Translational Medicine (Child Health), University of Liverpool, East Prescott Road, Liverpool L14 5AB, UK

7 Department of Pediatric Rheumatology, Alder Hey Children's NHS Foundation Trust Hospital, Liverpool, UK unpublished data on cytokine and chemokine expression in monocytes and tissues.

Summary $\mathrm{CNO} / \mathrm{CRMO}$ is an autoinflammatory bone disorder resulting from imbalanced cytokine expression from innate immune cells. Though the exact molecular pathophysiology remains unclear, variable molecular defects appear to result in inflammasome activation and pro-inflammatory cytokine expression in monocytes from $\mathrm{CNO} / \mathrm{CRMO}$ patients. Recent advances suggest signaling pathways and single molecules as biomarkers for $\mathrm{CNO} / \mathrm{CRMO}$ as well as future treatment targets.

Keywords Chronic non-bacterial osteomyelitis · CNO . Chronic recurrent multifocal osteomyelitis - CRMO . Treatment $\cdot$ Inflammation $\cdot$ Cytokine $\cdot$ Bone $\cdot$ Biomarkers

\section{Introduction}

Chronic non-bacterial osteomyelitis (CNO) is an autoinflammatory bone disorder mostly affecting children and adolescents [1-3]. Autoinflammatory disorders are characterized by an activation of the innate immune system in the absence of high-titer autoantibodies and (at least initially) no involvement of autoreactive lymphocytes. Several genetically inherited monogenic autoinflammatory conditions include early-onset non-infectious osteomyelitis, namely, Majeed syndrome, deficiency of interleukin-1 receptor antagonist (DIRA), and pyogenic arthritis, pyoderma gangrenosum, and acne syndrome (PAPA). Though sharing clinical and pathophysiological features with sporadic $\mathrm{CNO}$, this manuscript will only briefly discuss monogenic autoinflammatory bone disorders and mainly focus on CNO.

Sporadic CNO covers a wide clinical spectrum from rather mild, time-limited, monofocal bone inflammation to severe 
chronically active or recurrent multifocal bone inflammation. These most severe presentations are referred to as chronic recurrent multifocal osteomyelitis (CRMO). All ethnicities from all geographic regions can be affected. While highest disease incidences appear to exist in Western countries, particularly Central and Northern Europe, it remains unclear whether reporting issues may play a role, since no global epidemiologic studies have been performed. A genetic predisposition for $\mathrm{CNO}$ has been suggested by familial clusters of CNO patients [4-6] and associations with other inflammatory conditions, including inflammatory bowel disease, acne, ankylosing spondylitis, and psoriasis (Fig. 1) [1, 2, 7, 9-13]. In adults, patients with sporadic $\mathrm{CNO}$ are usually diagnosed with SAPHO, a symptom complex of synovitis, acne, pustulosis, hyperostosis, and osteitis $[9,10]$. Thus, SAPHO is currently seen as a closely related disorder with additional symptoms in the adult age group.

\section{Clinical Presentation and Epidemiology}

Clinical presentation and severity of $\mathrm{CNO}$ vary significantly between individual patients, covering a wide spectrum with asymptomatic or mild inflammation of single bones at the one end, and chronic recurrent multifocal, and sometimes bone destruction causing osteomyelitis at the other end (then referred to as chronic recurrent multifocal osteomyelitis, CRMO). CNO/CRMO most frequently involves metaphyses of long bones, the pelvic bones, the vertebral column, or the shoulder girdle/clavicle [3, 7, 12].

Clinical signs of bone inflammation include localized skin redness (rare), warmth and/or swelling, and pain. Additional

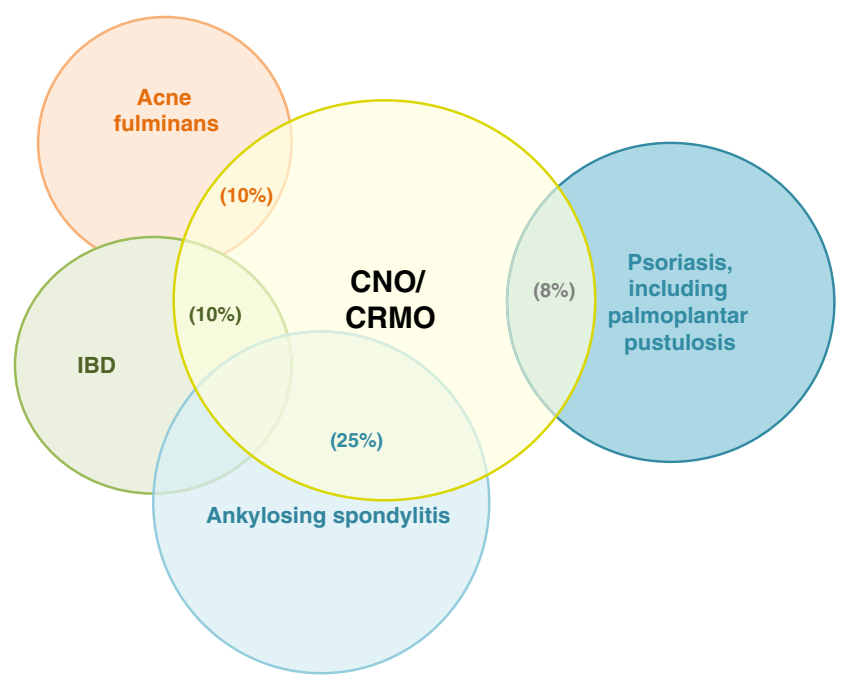

Fig. 1 Inflammatory organ involvement in $\mathrm{CNO} / \mathrm{CRMO}$. Psoriasis and palmoplantar pustulosis $(\sim 8 \%)$, inflammatory bowel disease $(\sim 10 \%)$, severe acne $(\sim 10 \%)$, and ankylosing spondylitis $(\sim 25 \%)$ have been demonstrated associated with $\mathrm{CNO} / \mathrm{CRMO}[7,8]$. (Figure modified after [8]) symptoms may be caused by paraosseous inflammation, involving peripheral nerves and/or vessels, skin or bowel inflammation, and synovitis. A subset of CNO patients exhibit inflammatory organ involvement, including psoriasis and palmoplantar pustulosis $(\sim 8 \%)$, inflammatory bowel disease $(\sim 10 \%)$, and severe acne $(\sim 10 \%)$ (Fig. 1$)$ [7]. Some CNO patients develop sacroiliitis, and some patients may progress from childhood CNO to spondylarthropathies in later life stages [14]. In adults, skin inflammation is significantly more common as compared to children. As mentioned above, acne and/or palmoplantar pustulosis frequently occur in the context of synovitis, hyperostosis, and osteitis, which is then referred to as SAPHO syndrome.

Epidemiological data in $\mathrm{CNO} / \mathrm{CRMO}$ are sparse, and include small case series and regional cohorts. CNO primarily affects children and adolescents, but can generally occur in all age groups. The peak onset of the disease is between 7 and 12 years of age [1-3, 9]. Chronic non-bacterial osteomyelitis is one of the most common autoinflammatory bone disorders in central Europe. According to several case series, CNO may be almost as common as infectious osteomyelitis [7, 15-17]. However, secondary to sometimes rather mild and unspecific clinical symptoms, CNO may be missed.

\section{Diagnostic Approach}

In the absence of widely accepted diagnostic criteria and disease biomarkers, $\mathrm{CNO} / \mathrm{CRMO}$ remains a diagnosis of exclusion. Clinical signs include bone pain, local swelling, rarely skin redness and heat, associated skin manifestations (including palmoplantar pustulosis, psoriasis, and acne), sometimes mildly elevated temperatures, and pathological fractures (usually of affected vertebral bodies). Non-infectious arthritis can be present in up to $30 \%$ of patients [3, 7]. Routine inflammatory parameters (WBC, white blood cell count; $\mathrm{CrP}, \mathrm{C}-$ reactive protein; ESR, erythrocyte sedimentation rate) are usually normal or mildly elevated.

Imaging techniques are centrally important for diagnosing $\mathrm{CNO} / \mathrm{CRMO}$ and excluding differential diagnoses [7]. Inflammatory bone lesions may be detected in plain radiographs as radiolucent, osteolytic, or sclerotic lesions [18-21], but may remain normal in early stages. Particularly in early disease, magnetic resonance imaging (MRI) techniques are highly sensitive. They can detect bone edema even before bone erosions and sclerosis develop, and help assessing inflammation of surrounding tissues. Strongly T2-weighted sequences (Turbo Inversion Recovery Measurement, TIRM) and/or gadolinium-enhanced T1 sequences with fat saturation are used to identify inflammatory bone lesions and/or periosseous affections [21-25]. At the time of diagnosis, whole body imaging using MRI techniques (TIRM) should be performed to identify clinically silent lesions, particularly 
in the vertebral column [24] (Fig. 2). MRI imaging techniques are also essential for the assessment of disease activity during follow-up, and the identification and monitoring of diseaseassociated sequelae, which may include fractures, inflammatory involvement and tissue damage to surrounding structures [24].

In unclear cases, bone biopsies are usually performed to exclude chronic infection, malignancies, or other systemic disease. Important differential diagnoses include malignancies (leukemia, lymphoma, primary and secondary bone tumors), infections (bacterial osteomyelitis, tuberculosis, etc.), immunodeficiency (e.g., defects in the IL-12: interferon axis that may be accompanied by mycobacterial infections), Langerhans cell histiocytosis ( $\mathrm{LCH})$, and other autoinflammatory disorders (e.g., Majeed syndrome [26-30], DIRA [31], or PAPA [9]). Disease onset before 2 years of age is extremely uncommon and should prompt considering differential diagnoses.

\section{Mechanisms of Chemokine and Cytokine Dysregulation in CNO/CRMO}

Despite recent scientific achievements, the exact molecular pathophysiology of CNO is only incompletely understood. Generally, familial (or monogenic) diseases including CNO as a descriptive symptom can be differentiated from the entity of sporadic $\mathrm{CNO}$, by other disease features. Though not the main focus of this manuscript, studies on monogenic/familial forms of "CNO" contributed to the pathophysiological understanding of sporadic $\mathrm{CNO} / \mathrm{CRMO}$.

\section{Familial/Monogenic CNO}

There are at least three human diseases involving chronic multifocal sterile osteomyelitis that are caused by single gene mutations: (i) Majeed syndrome (LPIN2 mutations), (ii) deficiency of interleukin-1 receptor antagonist (DIRA, mutations in $I L 1 R N)$ [ $26,32,33]$, and (iii) pyogenic arthritis, pyoderma gangrenosum, and acne syndrome (PAPA, mutations in PSTPIP1).

Majeed syndrome is characterized by early-onset multifocal osteomyelitis, dyserythropoietic anemia, and joint contractures. It is caused by loss of function mutations in the LPIN2 gene, encoding for the lipin 2 protein, a phosphatidate phosphatase (PAP) that plays a role in lipid metabolism. Lipin2deficient monocytes produce high amounts of proinflammatory cytokines IL- 6 and TNF $\alpha$ when stimulated by saturated fatty acids. Overexpression of LPIN2 on the other hand reduces inflammatory cytokine levels [34]. For Majeed syndrome, there is evidence that it is an IL- $1 \beta$-mediated disease, since bone inflammation and serum inflammation markers improve in response to IL-1 $\beta$ blockade, while TNF $\alpha$ blockers have almost no effect [35].

Deficiency of IL-1 receptor antagonist is characterized by early-onset destructive sterile bone lesions (osteitis and periostitis) and sterile pustulosis of the skin. If left untreated, DIRA leads to a severe systemic inflammatory response syndrome and respiratory failure [32] due to the lack of functional IL-1 receptor antagonist and subsequently uncontrolled IL-1 $\beta$ signaling. Treatment with recombinant IL-1 receptor antagonist results in prompt amelioration and disease control [32, 36].

Pyogenic arthritis, pyoderma gangrenosum, and acne are caused by mutation in the PSTPIP1 gene, involved in regulation of the actin cytoskeleton. PSTPIP1 binds to pyrin, a central negative regulator of the NLRP3 inflammasome. Therapeutic options for PAPA are local and/or systemic steroids, TNF $\alpha$ blockers, and IL-1 blocking agents [9, 37].

\section{"Sporadic" CNO/CRMO}

Genetic predisposition appears likely to be involved in the pathophysiology of "sporadic" CNO. It was suggested by rare familial clusters of $\mathrm{CNO} / \mathrm{CRMO}$ and high incidences of comorbid-affiliated inflammatory conditions such as psoriasis and inflammatory bowel disease in CNO patients and firstdegree family members (approximately 50\%) [7, 13]. Thus, the pathophysiology and inheritance of "sporadic" CNO appears to be complex with, e.g., a combination of associated risk alleles or individually variable (currently unknown) genetic causes resulting in disease resulting in clinically related phenotypes in the absence or presence of environmental factors.

A tight balance between pro- and anti-inflammatory signals is essential for immune homeostasis. Cytokines and chemokines play key roles in controlling inflammation and instructing immune responses. Consequently, dysregulation of their expression is linked with susceptibility to infectious and autoimmune diseases. It has become increasingly clear that appropriate temporal/spatial expression of cytokines and chemokines may be the key to the delicate balance between inflammation and immunoregulation. The mechanisms that govern the cell type- and receptor-specific induction of cytokines and chemokines, however, in most instances remain unclear [38, 39]. Our current pathophysiological understanding of $\mathrm{CNO} / \mathrm{CRMO}$ is based on a profound imbalance between pro- and anti-inflammatory cytokines $[40,41 \bullet \bullet, 42$, 43] (Fig. 3). We demonstrated that monocytes from CRMO patients fail to express the immune regulatory IL-10 in response to Toll-like receptor (TLR) 4 stimulation with lipopolysaccharide (LPS) [42, 43]. Impaired IL-10 expression is (at least partially) caused by reduced activation of mitogenactivated protein kinases (MAPK), ERK1 and 2 [42], resulting in impaired activation and nuclear shuttling of the 

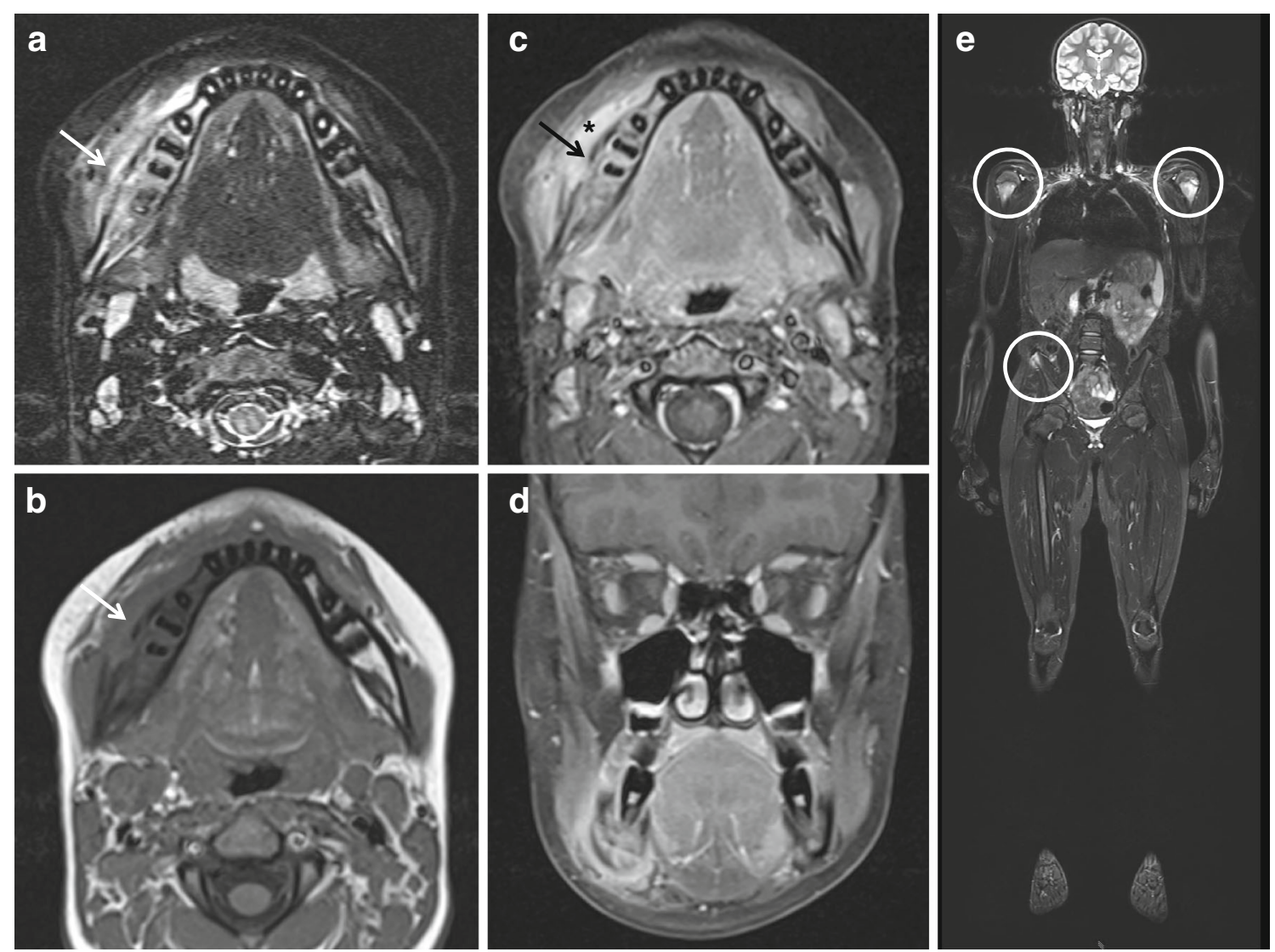

Fig. 2 Magnetic resonance imaging in $\mathrm{CNO} / \mathrm{CRMO}$. Magnetic resonance imaging in a 15-year-old male patient with swelling and warmth over right mandible. a Transversal TIRM sequences unveiled bone swelling and edema of the right mandible (arrow). b Native and $\mathbf{c}$ contrast-enhanced transversal $\mathrm{T} 1$ sequences with fat saturation unveiled new bone formation (arrows), resulting swelling, and in $\mathbf{c}$ enhancement in the right mandible

transcription factor signaling protein (SP-)1, and subsequently altered recruitment of Sp-1 to the IL10 promoter. Furthermore, attenuated ERK activation results in reduced histone H3S10 phosphorylation at the IL10 promotor [42, 43], an activating epigenetic modification. Disturbed epigenetic remodeling and reduced transcription factor binding to the $I L 10$ promoter result in impaired IL-10 expression and a disruption of balanced pro- and anti-inflammatory cytokine expression.

Together with its homologs $I L 19$ and $I L 20$, the $I L 10$ gene is organized in the so-called IL10 cytokine cluster on chromosome 1q32. IL-10 and IL-19 mostly bear immune regulatory functions, while IL-20 exerts pro-inflammatory properties [41••]. Thus, we asked whether the expression of IL-19 and IL-20 are also altered in monocytes from CNO/CRMO patients. In analogy to IL10, IL19 is regulated by Sp-1 and epigenetic remodeling which are altered in monocytes from CRMO patients (H3S10 phosphorylation, DNA methylation), contributing to reduced IL-19 expression in monocytes from CRMO patients [41••].

Notably, the expression of pro-inflammatory IL-20 was not reduced. Contrary, after LPS stimulation, IL-20 expression (asterisk). d Coronary T1 sequences with fat saturation in the same patient. e Whole body MRI (coronary TIRM sequences) unveiled additional sites of bone inflammation at both proximal humeri and the right upper iliac spine (circles) (MRI images with friendly permission from Gabriele Hahn, Pediatric Radiology, Medizinische Fakultät Carl Gustav Carus, Technische Universität Dresden, Dresden, Germany)

Fig. 3 Molecular pathophysiology of CNO/CRMO. Inflammation is a potent and undirected defense mechanism against exogenous pathogens or endogenous danger signals (such as infections, tissue damage). The sensing of danger signals occurs by pattern recognition receptors (PRRs), such as the membrane-associated Toll-like receptors (TLRs) and the predominantly cytoplasmic localized NOD-like receptors (NLRs). After recognition of danger signals by monocytes/macrophages, multiprotein complexes, referred to as inflammasomes are activated. The NLRP3 inflammasome comprises NLRP3, ASC, and procaspase-1. After inflammasome activation, caspase- 1 cleaves pro-IL- $1 \beta$ and leads to the secretion of active IL- $1 \beta$. In monocytes from CRMO patients, MAP kinase Erk1 and 2 signaling is impaired, resulting in reduced expression of the immune regulatory cytokines IL-10 and IL-19. JNK and p38 MAPK are unaffected, leading to the expression of pro-inflammatory cytokines (TNF $\alpha$, IL- 6 , IL-1 $\beta$, IL-20). Reduced expression of IL-10 and IL-19 contributes to increased inflammasome activation and subsequent IL-1 $\beta$ release. Pro-inflammatory cytokines TNF $\alpha$, IL-6, IL-20, and IL- $1 \beta$ increase the interaction of membrane RANK receptors with their soluble ligand RANKL on osteoclast precursor cells and induce osteoclast differentiation and activation.MAPK: mitogen-activated protein kinase; CRMO: chronic recurrent multifocal osteomyelitis; Erk1: extracellular signal-regulated kinase-1; TLR: Toll-like receptor; IL: interleukin; JNK: Jun kinase; TNF: tumor necrosis factor; NF- $\mathrm{BB}$ : nuclear factor- $\mathrm{B}$; Casp1: caspase-1; PAMP: pathogen-associated molecular pattern; DAMP: danger-associated molecular pattern; RANK: receptor activator of nuclear factor- $\mathrm{kB}$; RANKL: RANK ligand 


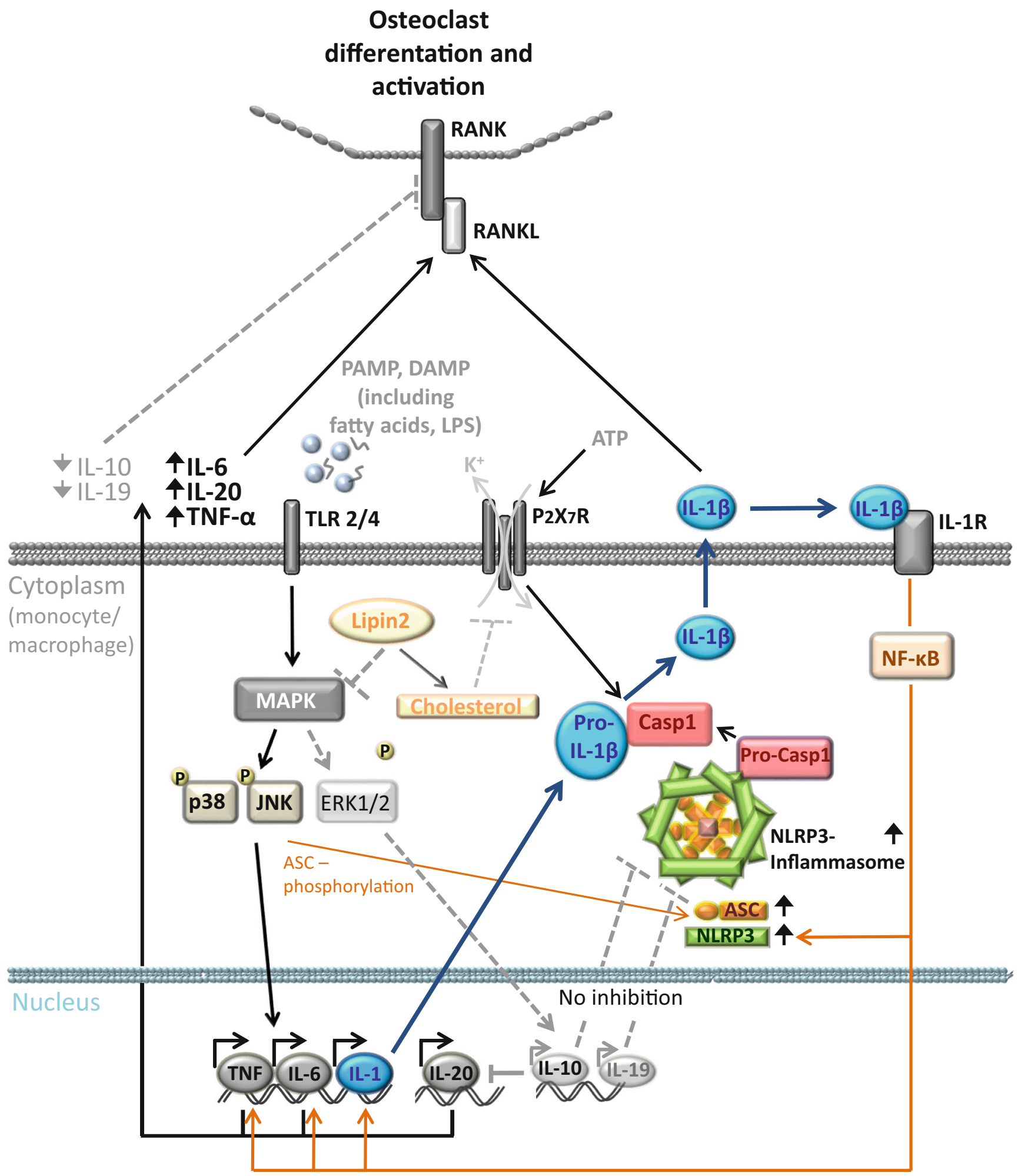

was enhanced in monocytes from CRMO patients, likely due to reduced DNA methylation of IL20 [41••]. The expression of other MAPK-induced pro-inflammatory cytokines including TNF $\alpha$ and IL- 6 was not reduced but rather increased in monocytes from CRMO patients (Fig. 4b). This may at least partially be explained by the fact that the alternative p38 MAPK pathway was not affected [42].

Recently, inflammatory bone loss and synovial inflammation in IL-10-deficient mice were linked to NLRP3 inflammasome activation [44•]. Furthermore, Scianaro et al. 

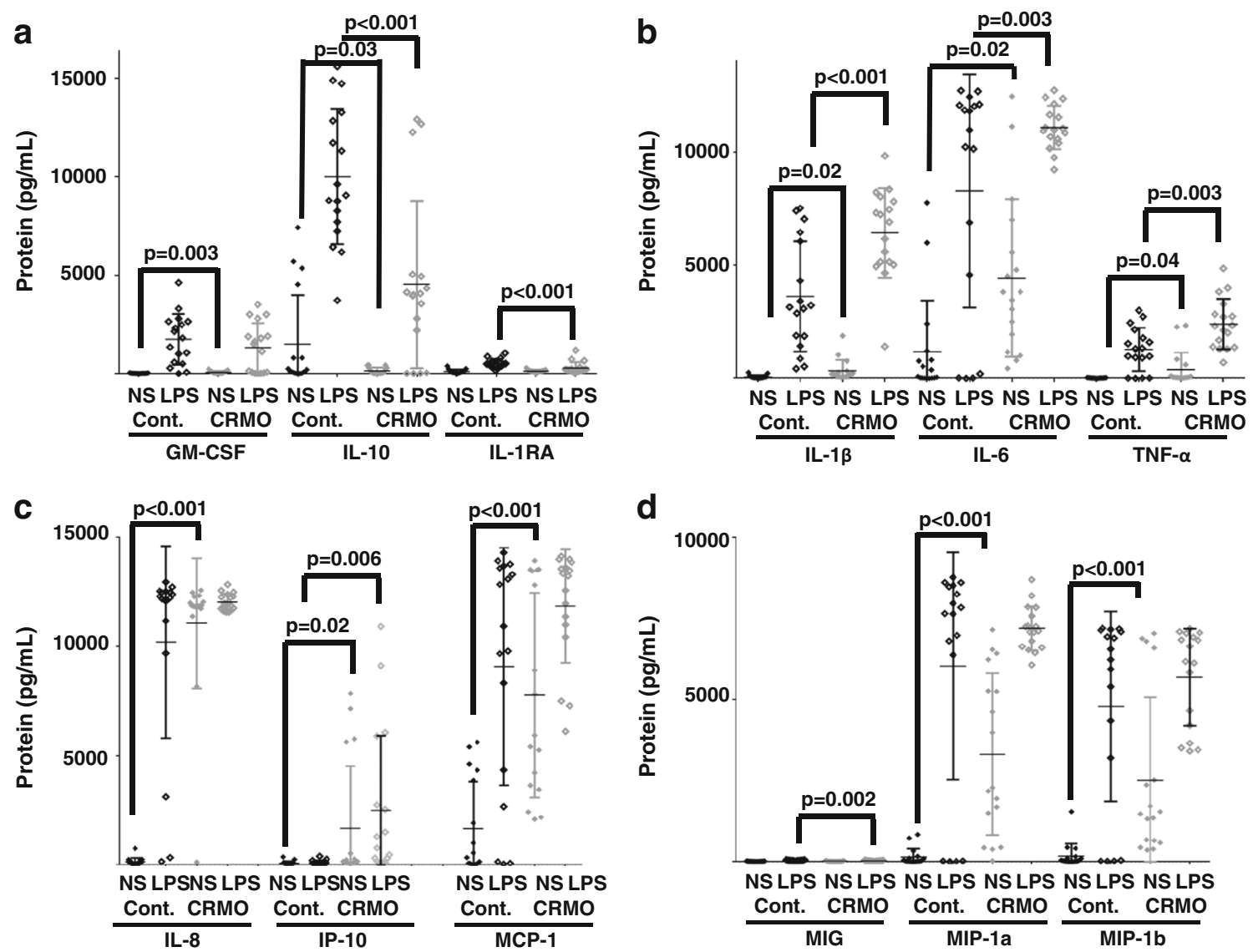

Fig. 4 Inflammation marker expression in monocytes from CRMO patients. Monocytes from treatment-naive CRMO patients $(N=17)$ and age and gender matched healthy controls $(N=17)$ were isolated by negative selection of $\mathrm{CD}^{+} 4^{+}$cells using standard procedures (Miltenyi). Monocytes were cultured at $1 \times 10^{6}$ per milliliter media (RPMI, gentamycine, penicillin) with $5 \% \mathrm{CO} 2$ at $37{ }^{\circ} \mathrm{C}$. Some cells were stimulated with $100 \mathrm{ng} / \mathrm{mL}$ LPS over night as indicated. Protein levels were measured from cell culture supernatants on the LUMINEX2000 platform, using multiplex gene expression arrays (Millipore). a Monocytes from CRMO patients fail to express immune regulatory proteins GM-CSF (under resting conditions), IL-10 (under resting conditions and after stimulation with LPS), and IL-1RA (in response to

[45] suggested increased NLRP3 inflammasome activation contributing to the inflammatory phenotype in CRMO, showing increased mRNA expression of inflammasome components (ASC, NLRP3, caspase-1) as well as increased IL-1 $\beta$ transcription and release from peripheral blood mononuclear cells from active CRMO patients compared to patients with inactive disease and controls after stimulation with LPS. We recently linked impaired IL-10 and IL-19 expression with increased IL- $1 \beta$ mRNA expression and IL- $1 \beta$ release in monocytes from CRMO patients [41••]. Enhanced inflammasome activation and IL- $1 \beta$ secretion by monocytes from CRMO patients is reversible by co-culture with recombinant IL-10 or IL-19 [41••], suggesting an immunomodulatory function of IL-10 and IL-19 on inflammasome activation.

These observations resulted in the hypothesis that imbalanced expression of anti- (IL-10 and IL-19) and pro-

stimulation with LPS). b Monocytes from CRMO express increased levels of pro-inflammatory IL-1b, IL-6, and TNF $\alpha$ (under resting conditions and in response to stimulation with LPS). $\mathbf{c}$ and $\mathbf{d}$ Monocytes from CRMO patients express increased amounts of proinflammatory chemokines IL-8, MCP-1, MIP-1a, and MIP-1b under resting conditions, MIG in response to stimulation with LPS, and IP-10 under resting conditions and in response to stimulation with LPS. These observations further indicate a significant imbalance in the expression of pro- and anti-inflammatory proteins, suggesting a pro-inflammatory phenotype of monocytes in CRMO which show signs of "spontaneous" activation

inflammatory cytokines (IL-1, IL-6, TNF $\alpha$, IL-20) may result in increased osteoclast differentiation and activation through enhanced interaction between receptor activator of nuclear factor- $\mathrm{KB}$ (RANK) and its soluble ligand RANKL on osteoclast precursor cells (Fig. 3) [9, 46, 47].

In addition to the molecular mechanisms mentioned above, IL-10 expression is predetermined by genetic variants within the IL10 proximal promoter region. Three promoter haplotypes rs1800896, rs1800871, and rs1800872, resulting in three haplotype blocks (GCC, ACC, and ATA), influence the capacity of the $I L 10$ promoter to recruit the transcription factor Sp-1. Interestingly, and to our initial surprise, in cohorts of CRMO patients, IL10 promoter haplotype blocks encoding for "high" IL-10 expression (GCC) were significantly more common when compared to such encoding for "low" gene expression (ATA) [48]. Provided the molecular pathologic 
mechanisms discussed above, we hypothesized that individuals with CRMO-associated molecular disturbances and IL10 promoter haplotype blocks encoding for "low" IL-10 expression may develop more severe disease and may not be diagnosed with $\mathrm{CNO} / \mathrm{CRMO}$ but other inflammatory conditions. However, this hypothesis remains to be confirmed scientifically.

Recently, a CRMO susceptibility gene has been identified through whole exome sequencing and gene expression microarrays [49]. One homozygous and one compound heterozygous mutation in the filamin-binding domain of the FBLIMI gene were detected in unrelated CNO patients from South Asia [49]. Filamin-Binding LIM Protein 1 (FBLIM1) has been suggested to act as an anti-inflammatory molecule that controls bone remodeling through the regulation of RANKL activation via ERK1/2 phosphorylation [49]. On the transcriptional level, FBLIM1 expression is regulated by the transcription factor STAT3 [49]. Since the immune regulatory cytokine IL-10 induces STAT3 activation, aforementioned haplotype blocks within the IL10 promoter may be involved in the pathophysiology of CNO. Indeed, Cox et al. demonstrated that both individuals carried such IL10 promoter haplotypes that code for "low" IL-10 expression, which may in turn contribute to reduced STAT3 activation and resulting effects on FBLIM1 expression in the reported individuals $[50 \bullet \cdot$.

\section{Biomarkers for the Diagnosis and Monitoring of CRMO}

Currently, widely accepted disease biomarkers for the diagnosis of CNO/CRMO are not available. Recently, we reported a preliminary set of serum inflammatory parameters that allow differentiating between newly diagnosed and treatment naïve patients with CRMO, Crohn's disease, and healthy controls. Biomarkers included the monocyte derived chemokines monocyte chemotactic protein (MCP-)1 and macrophage inflammatory protein (MIP-)1b, the pro-inflammatory cytokines IL-6 and IL-12, the mast cell derived chemokine eotaxin, RANTES, the soluble IL-2 receptor, and the IL-1 receptor antagonist. Increased serum levels of the inflammatory cytokines IL-6 and IL-12, the chemokines MCP-1, MIP-1b, RANTES, and eotaxin, and the soluble IL-2 receptor distinguished among CRMO patients, individuals with Crohn's disease and healthy controls. However, the proposed set of biomarkers could not distinguish between patients with CRMO or ANA-positive, HLA B27-negative juvenile idiopathic arthritis (JIA). This may be caused by pathophysiological parallels between the two disorders and/or a currently incomplete set of tested parameters [51]. Currently, follow-up studies are under way, including additional parameters and differential diagnoses.

In addition to serum biomarkers, cytokine and chemokine expression from isolated immune cells may be used to diagnose CNO/CRMO. In Fig. 4, cytokine and chemokine expression patterns from ex vivo isolated peripheral blood monocytes from CRMO patients and controls are provided. Indeed, monocytes from CRMO patients fail to express granulocyte monocyte colony-stimulating factor (GM-CSF), and the anti-inflammatory molecules IL-10 and IL-1 receptor antagonist (IL-1RA) under resting conditions and/or in response to TLR4 stimulation with LPS (Fig. 4a). Conversely, monocytes from CRMO patients express increased amounts of proinflammatory cytokines (IL-1 $\beta$, IL-6, TNF $\alpha$; Fig. $4 \mathrm{~b}$ ) and chemokines (IL-8, Interferon gamma-induced protein 10: IP10, MCP-1, MIG, MIP-1a, MIP-1b; Fig. 4c, d) in most cases already under resting conditions. These findings support previous reports on dysbalanced cytokine and chemokine expression and promise potential as disease biomarkers for the diagnosis of CNO/CRMO [41••, 42, 43, 51]. However, additional studies are needed to confirm findings, to extend beyond included differential diagnoses, and to generate longitudinal data sets measuring treatment responses.

Lastly, gene expression profiles in situ may be used as disease biomarkers. Preliminary studies in bone biopsies from patients with CRMO, Langerhans cell histiocytosis (LHC), bacterial osteomyelitis (BOM), or from healthy controls delivered promising results. In agreement with the aforementioned observations in ex vivo isolated monocytes, IL-10 expression in bone tissue from CRMO patients appears reduced when compared to other inflammatory conditions (LHC and BOM; Fig. 5). Expression of the inflammasome component NLRP3 and the pro-inflammatory cytokine IL- $1 \beta$ is increased in samples from CRMO patients when compared to LHC patients or healthy controls. Patients with BOM, however, (not surprisingly) exhibit even higher inflammasome activation and IL-1 $\beta$ expression levels when compared to CRMO (Fig. 5).

\section{Murine Models of CRMO}

Studies in primary human cells and in cell lines are limited by intrinsic and/or extrinsic factors, including sample sizes, availability (in primary human cells), culturing conditions, and biological limitations in immortalized cells. Several murine models are available to study underlying pathologic mechanisms of non-infectious bone inflammation. Mice deficient of proline-serine-threonine phosphatase-interacting protein 2 spontaneously develop bone inflammation, elevated proinflammatory cytokines in the blood, extramedullary hematopoiesis and skin inflammation, resembling very severe CRMO (Table 1).

Lupo mice carry a chemically induced homozygous mutation (c.Y180C; p.I282N) in the proline-serine-threonine phosphatase-interacting protein 2 (Pstpip2) gene [52, 53]. Chronic multifocal osteomyelitis $(\mathrm{cmo})$ mice carry a spontaneously acquired homozygous mutation (c.T293C, p.L98P) in 

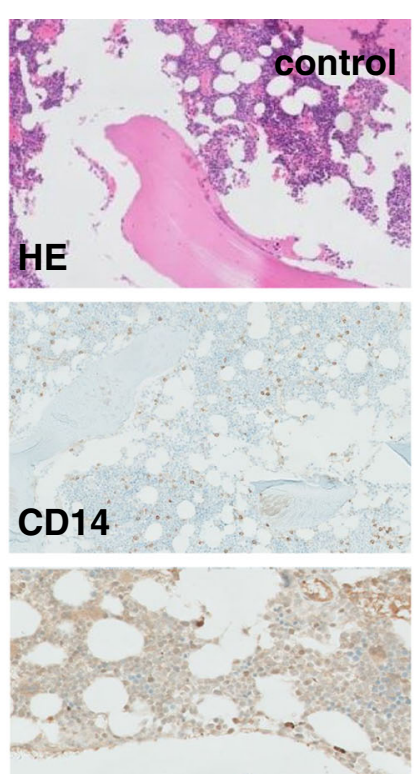

\section{NLRP3}
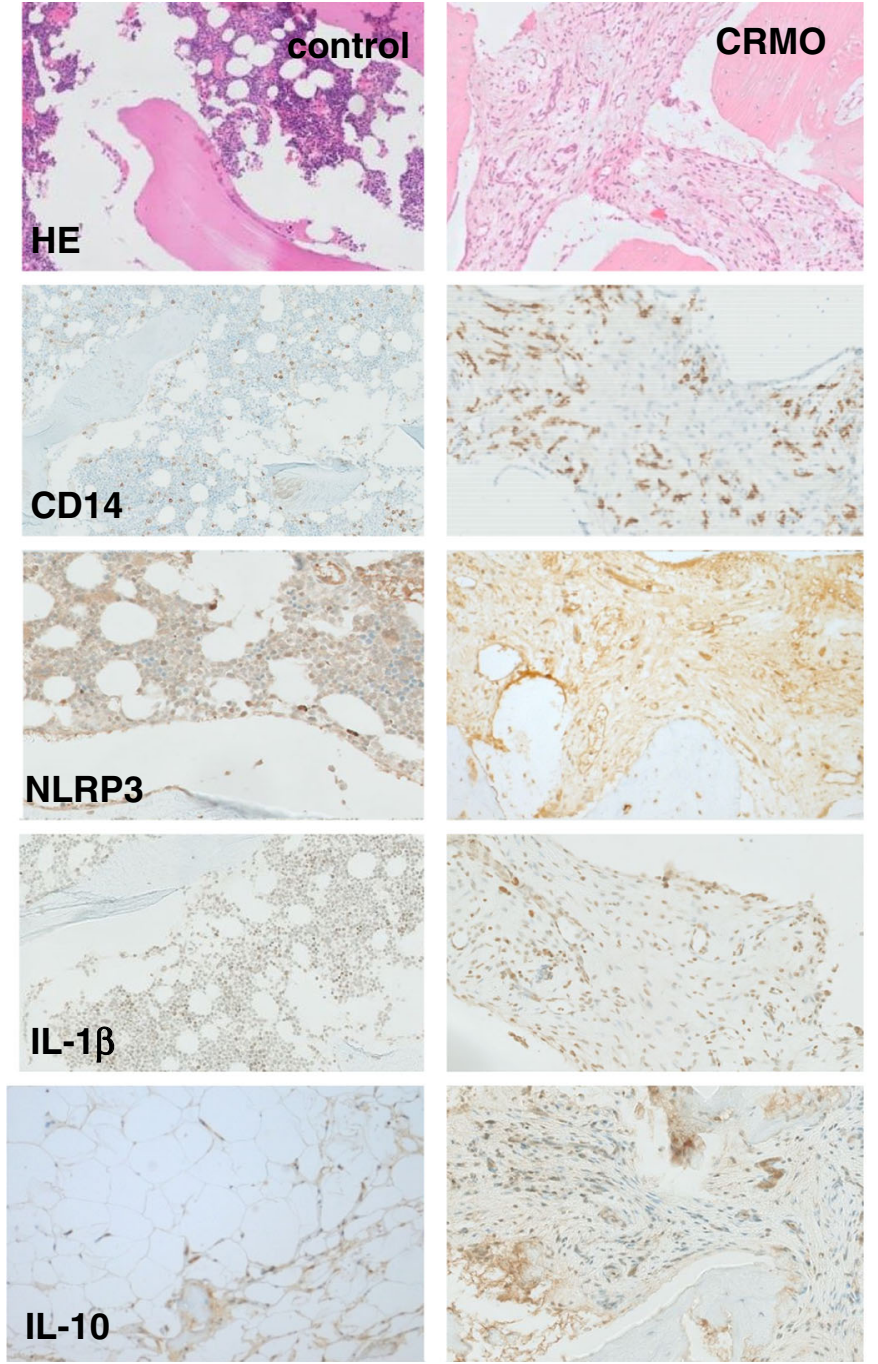

Fig. 5 Histomorphological appearance of bone inflammation in $\mathrm{CNO} /$ CRMO, bacterial osteomyelitis (BOM), and Langerhans cell histiocytosis (LCH). Formalin-fixed, decalcified, and paraffin-embedded bone biopsy specimens were immune-stained with antibodies directed against CD14 (monocyte marker), NLRP3, IL-1 $\beta$, and IL-10 using standard techniques (as indicated). Displayed magnification is $\times 100$. In the top panel, HE stains are displayed. Control: trabecular bone with fatty marrow and hematopoietic tissue from a bone healthy patient undergoing osteotomy. CRMO: moderately dense infiltrate of inflammatory cells, predominantly neutrophils and monocytes, some marrow fibrosis. BOM: dense infiltrate of inflammatory cells with predominant neutrophils, cellular bone
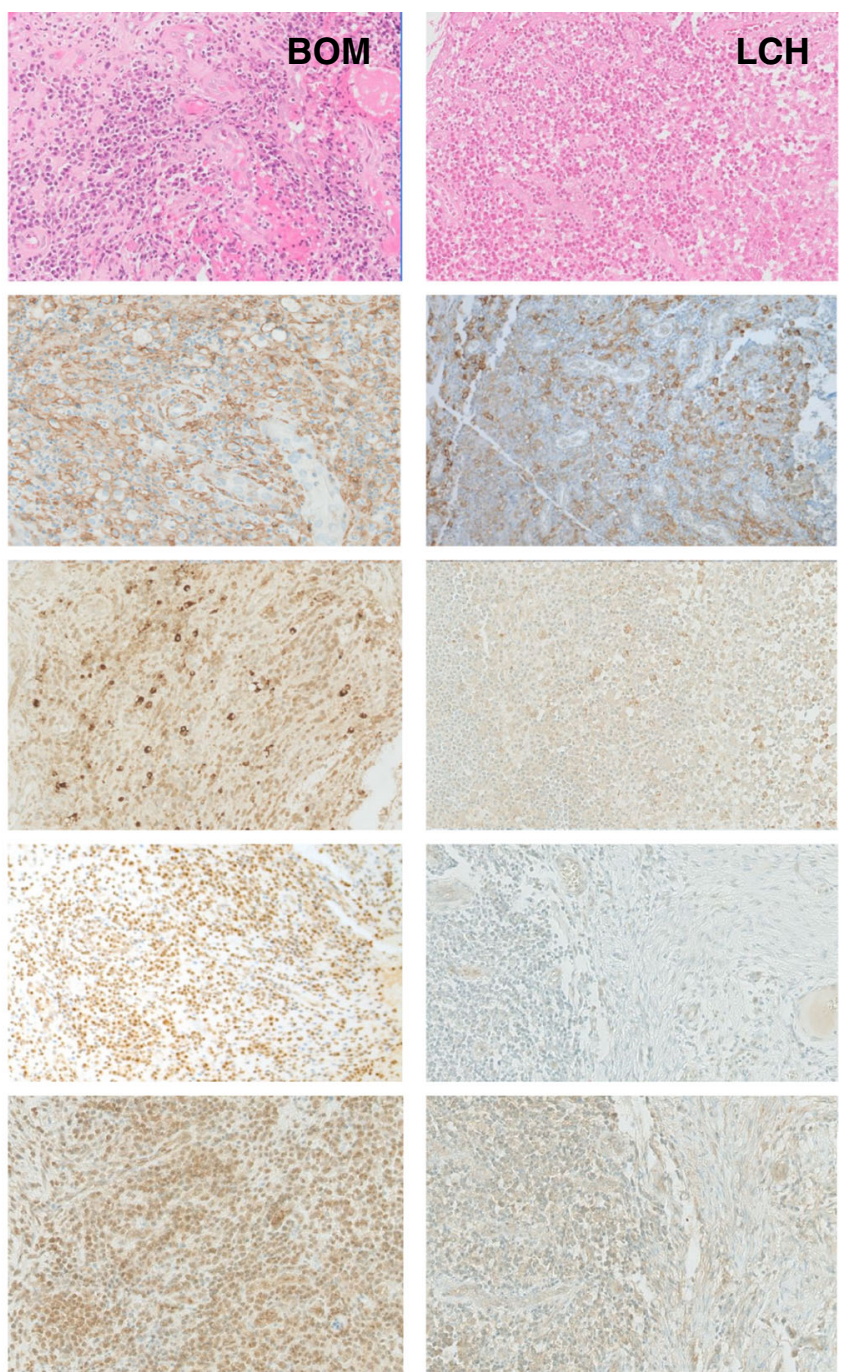

remodeling and bone necrosis. LCH: Ovoid Langerhans cells, some with linear grooves of nuclei, admixed with inflammatory cells, including a large number of eosinophils, lymphocytes, neutrophils, and plasma cells. Infiltrates of CD14-positive monocytes are a central component of inflammation in acute BOM, early phase CRMO, and LCH. Expression of the inflammasome component NLRP3 is increased in inflammatory infiltrates of BOM $>$ CRMO $>$ LOM, translating into IL$1 \beta$ protein expression in BOM and CRMO. As suggested by studies in ex vivo isolated monocytes, IL-10 expression in inflammatory bone lesions from CRMO patients is reduced as compared to lesions from $\mathrm{BOM}$ and $\mathrm{LCH}$ patients

Table 1 Pstpip2 mutant mice strains as disease models for CRMO. cmo chronic multifocal osteomyelitis, ENU N-ethyl-N-nitrosourea, Pstpip2 proline-serine-threonine phosphatase-interacting protein 2

\begin{tabular}{llll}
\hline Pstpip2 mutant mice & Generation & Mutation & References \\
\hline lupo mice & ENU mutagenesis, homozygous mutation & p.I282N & c.Y180C \\
cmo mice & Homozygous spontaneous mutation & p.L98P & [52, 53] \\
Targeted knockout & Conventional knockout & Target exons 3 and 4 & {$[26,54,28,55,56]$} \\
& Conditional knockout & & {$[57]$} \\
\hline
\end{tabular}


Pstpip2. To date, the exact molecular contribution of Pstpip2 mutations to sterile bone inflammation remains somewhat unclear [57]. Pstpip2 belongs to the F-BAR (Fes/CIP4 homology-Bin/Amphiphysin/Rvs) domain containing protein superfamily, which couples membrane remodeling with actin dynamics associated to endocytic pathways and filopodium formation [58]. It is a cytosolic, cytoskeleton-associated adapter molecule, which interacts with formin binding protein 17 (FBP17) through its F-BAR domain. In the presence of Pstpip2, an antagonistic recruitment of FBP17 and Pstpip2 to the plasma membrane enables correct activation of actin polymerization at podosomes. In the absence of Pstpip2, actin polymerization is hyperactivated by constitutive membrane recruitment of the FBP17-WASP (Wiskott-Aldrich syndrome protein) complex [59]. Macrophages that express Pstpip2 at reduced levels exhibit abnormal podosome formation, leading to a more invasive phenotype.

Interleukin-1 $\beta$ has been linked with the pathogenesis of osteomyelitis in cmo mice [60,61••]. Cmo mice lacking the functional IL-1 receptor I (IL-1RI) or IL-1 $\beta$ (but not IL-1 $\alpha$ ) were completely protected from $\mathrm{CNO}[60,61 \bullet \cdot]$. Conversely, cmo mice deficient of the inflammasome components
NLRP3, ASC or caspase-1 developed severe CNO, indicating that there must be another kinase or protease other than caspase-1-activating IL-1 $\beta$. Previously described proteases, performing IL- $1 \beta$ cleavage includes neutrophil serine proteases or caspase-8 [62・•], suggesting that neutrophils may play a central role in disease pathogenesis. Thus, Cassel et al. performed experiments to identify immune cell subsets critical for IL-1 $\beta$ release in cmo mice [60]. LPS-primed and ATPstimulated bone marrow cells but not bone marrow derived macrophages from cmo mice produced high amounts of IL$1 \beta$. Increased IL-1 $\beta$ production was reduced by treatment of cmo bone marrow with a serine protease inhibitor (diisopropylfluorophosphate) but not with the pan-caspase-1 inhibitor z-YVAD-fmk [60], suggesting the involvement of neutrophils in the pathogenesis of $\mathrm{cmo}$. Findings were confirmed by Lukens et al. [61••], who additionally showed that pharmacological depletion of neutrophils with the monoclonal antibody anti-Ly6G protected cmo mice from CNO [63•]. Interestingly, сmo mice either deficient of caspase-1 or -8 developed $\mathrm{CNO}$, whereas cmo mice deficient of both caspases were protected from disease [63•], indicating that both caspases play redundant roles in $\mathrm{cmo}$ mice.

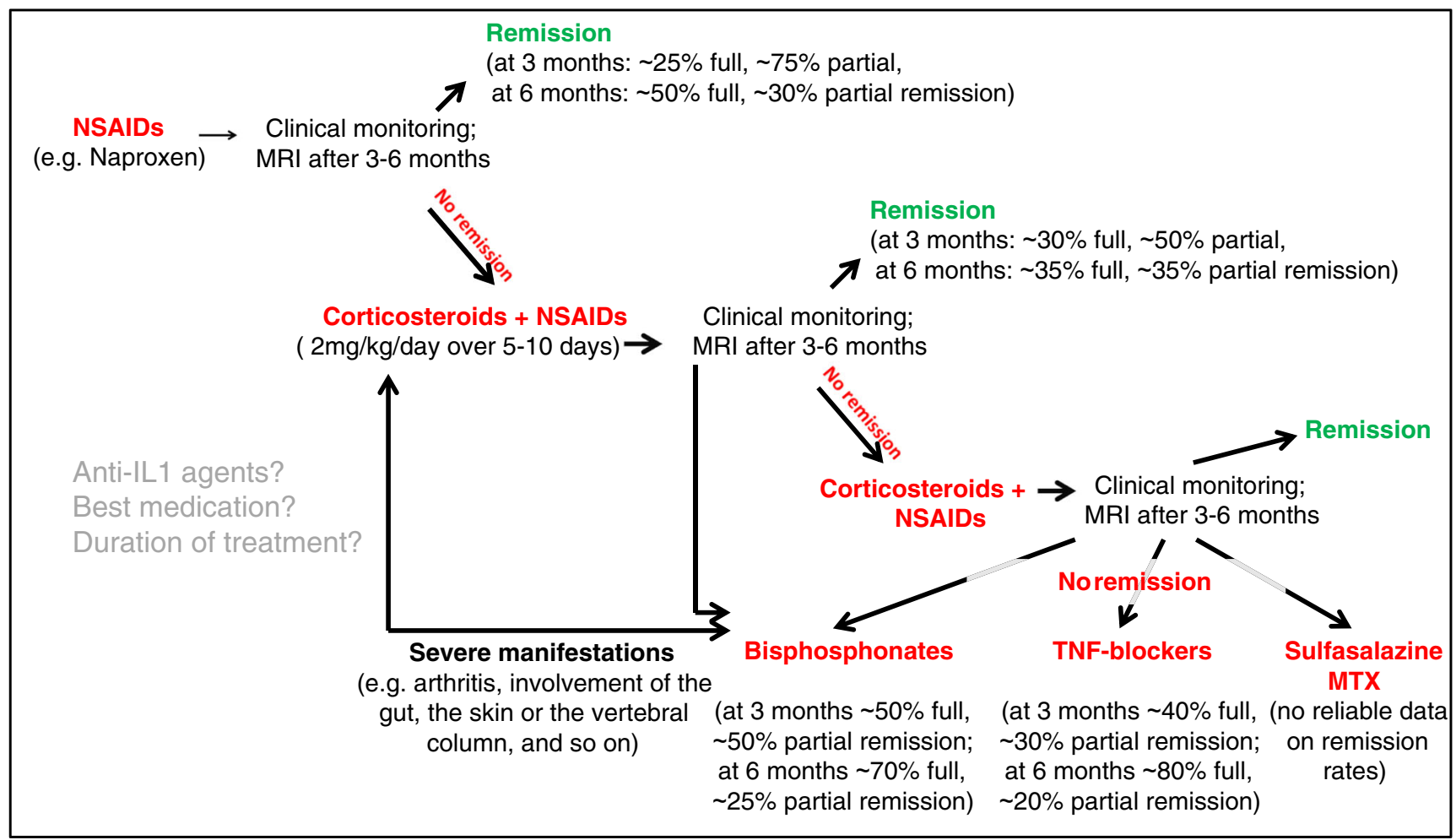

Fig. 6 Treatment of CNO/CRMO. NSAIDs (e.g., naproxen) are usually applied as first-line therapy in $\mathrm{CNO} / \mathrm{CRMO}$ patients. Monitoring includes clinical investigations and MRI scans after 3 to 6 months. Treatment goals are clinical and, in the case of vertebral involvement, radiological remission. In NSAID-refractory cases, treatment can be escalated with corticosteroids. The authors usually apply $2 \mathrm{mg} / \mathrm{kg}$ oral prednisone per day plus NSAIDs over 5 to 10 days. In cases who first respond to corticosteroid treatment but then flare, high-dose steroids ( $2 \mathrm{mg} / \mathrm{kg} / \mathrm{day})$ can be repeated and supplemented by low-dose corticosteroids $(0.1-$ $0.2 \mathrm{mg} / \mathrm{kg} /$ day) over a longer period, e.g., to "bridge" until DMARDs are working. In individuals who fail to reach clinical and (if vertebrae are involved) radiological remission or relapse again, bisphosphonates, $\mathrm{TNF} \alpha$ inhibitors, sulfasalazine, or methotrexate (MTX) should be considered. In patients with vertebral body involvement and structural damage, aggressive treatment should be discussed initially, e.g., with bisphosphonates 
Taken together, in agreement with aforementioned findings in monocytes from CRMO patients, recent work in cmo mice suggests a central involvement of IL- $1 \beta$ in disease pathophysiology. Complete deficiency of Pstpip 2 results in dysregulated production of IL-1 $\beta$ by neutrophils and enhanced osteoclastogenesis. Of note, myeloid cells determine the phenotype in Pstpip2-deficient animals (at least initially) independent of the adaptive immune system [54].

Over the recent years, it became increasingly clear that host interactions with skin and gut microbiota have significant effects on immune homeostasis [64]. Particularly in genetically predisposed individuals, alterations to microbiomes can result in uncontrolled inflammation and the expression of autoimmune/inflammatory conditions. Recent data from Lukens et al. [63•] suggest that dietary manipulation of the microbiome in cmo mice can prevent osteomyelitis. The exact molecular mechanisms involved, however, remain unclear. Patients with CRMO exhibit associations with severe acne $(10 \%)$ and inflammatory bowel disease (10\%) [7], which underscores the therapeutic potential of these observations, since all of these CRMOassociated disorders are characterized by significant alterations to microbiomes [64].

\section{Treatment and Monitoring}

Treatment of patients with $\mathrm{CNO} / \mathrm{CRMO}$ is largely based on expert opinion and relatively small case collections. It usually involves non-steroidal anti-inflammatory drugs (NSAIDs), corticosteroids, disease-modifying anti-rheumatic drugs (DMARDs, usually methotrexate or sulfasalazine), anti-TNF agents, or bisphosphonates (Fig. 6).

Several retrospective analyses and one prospective observation indicate that NSAIDS are effective in a large subset of patients within the first 1-2 years of treatment. We, however, recently documented that more than $50 \%$ of patients flare after 2 years [7, 65 $]$. Corticosteroids appear to quickly and effectively control inflammatory activity, but rarely induce longterm remission. Though only reported in a small number of patients, treatment with pamidronate or anti-TNF agents has been reported to be highly effective in a significant percentage of $\mathrm{CNO} / \mathrm{CRMO}$ patients, inducing long-lasting remission in a large subset of patients $[3,9,66]$. However, all reports are limited by retrospective data collection and relatively small patient numbers.

Large prospective clinical trials to determine the best medication and the duration of treatment are lacking. Currently, consensus treatment plan initiatives of the North American Childhood Arthritis and Rheumatology Research Alliance (CARRA) and the German Society of Pediatric Rheumatology (GKJR) are under way and will investigate and compare treatment responses to currently used therapeutic agents and protocols.

In addition to the currently applied treatment options, blockade of other molecules may be beneficial in $\mathrm{CNO}$ / CRMO. Since IL-1 $\beta$ is involved in the molecular pathophysiology of CNO/CRMO, recombinant IL-1 receptor antagonist anakinra or anti-IL-1 antibody treatment with canakinumab may be applied. Furthermore, IL-1 blockade proved effective in familial monogenic autoinflammatory disorders involving CNO (namely, Majeed syndrome and DIRA) [32, 35]. However, surprisingly few cases of antiIL-1 treatment have been reported and showed mixed response with variable outcomes. The potential explanation for the poor response may include low tissue concentrations, pathophysiological heterogeneity in CNO/CRMO, among others.

Blockade of RANKL with the recombinant RANK ligand inhibitor denosumab may reduce osteoclast activation and inflammatory bone loss in $\mathrm{CNO} / \mathrm{CRMO}$ and promises potential in the treatment of $\mathrm{CNO} / \mathrm{CRMO}$. However, at this point, there are no reliable reports on successful application of these treatment options in CNO/CRMO.

Monitoring disease activity is a concern in $\mathrm{CNO} / \mathrm{CRMO}$ patients, since a significant subset of patients may develop pain amplification syndrome. Thus, clinical scores including pain scores, routine inflammatory parameters, and imaging results have been suggested (PedCNO score) [66]. However, to date, scores are incompletely evaluated and involve time consuming and costly investigations (e.g., MRI). Thus, easily accessible and inexpensive disease biomarkers for the assessment of disease activity are urgently warranted. We recently reported a preliminary set of biomarkers (IL-12, MCP-1, sIL-2R) that may act as markers for treatment response to NSAIDs [51]. Though included as a parameter in PedCNO scores, ESR and CrP correlated less closely with PedCNO scores when compared to the reported serum biomarkers. Thus, after further evaluation in independent cohorts and in response to other treatment options, IL-12, MCP-1, and/or sIL-2R may be used as future biomarkers for disease activity in $\mathrm{CNO} / \mathrm{CRMO}$.

\section{Conclusions}

Chronic non-bacterial osteomyelitis with its most severe form chronic recurrent multifocal osteomyelitis is an inflammatory bone disorder that can result in damage to bones and other tissues. Due to the lack of widely accepted diagnostic criteria or disease biomarkers, $\mathrm{CNO} / \mathrm{CRMO}$ remains a diagnosis of exclusion. The molecular pathophysiology of CNO/CRMO remains incompletely understood. However, dysregulated cytokine expression from innate immune cells centrally contributes to the inflammatory phenotype of CNO. Treatment is 
largely based on small, mostly retrospective case collections and expert opinion, and prospective studies are largely lacking. Currently, consensus treatment protocols are under way and will deliver reliable data on treatment responses and outcomes. The recent identification of several genetic and molecular alterations in $\mathrm{CNO} / \mathrm{CRMO}$ promise future success in determining exact pathophysiological causes and target-directed treatment options.

Acknowledgements Sigrun Hofmann was supported by German Research Foundation (DFG) Grant KFO249, TP2, HO4510/1-2, and Christian Hedrich was supported by the Thyssen Foundation, the intramural MeDDrive program of TU Dresden, the Else Kröner Foundation, and the Foundation for Therapeutic Research.

\section{Compliance with Ethical Guidelines}

Conflict of Interest Jessica Pablik, Sigrun Hofmann, Herman Girschick, Polly Ferguson, Franz Kapplusch, Henner Morbach, and Christian Hedrich declare no conflict of interest.

Human and Animal Rights and Informed Consent Studies in human tissue samples and primary human cells were approved by the local ethical committees by TU Dresden and University of Wuerzburg. Individuals or their legal guardians gave written informed consent.

Open Access This article is distributed under the terms of the Creative Commons Attribution 4.0 International License (http:// creativecommons.org/licenses/by/4.0/), which permits unrestricted use, distribution, and reproduction in any medium, provided you give appropriate credit to the original author(s) and the source, provide a link to the Creative Commons license, and indicate if changes were made.

\section{References}

Papers of particular interest, published recently, have been highlighted as:

- Of importance

•- Of major importance

1. Hedrich CM, Hahn G, Girschick HJ, Morbach H. A clinical and pathomechanistic profile of chronic nonbacterial osteomyelitis/ chronic recurrent multifocal osteomyelitis and challenges facing the field. Expert Rev Clin Immunol. 2013;9:845-54.

2. Stern SM, Ferguson PJ. Autoinflammatory bone diseases. Rheum Dis Clin N Am. 2013;39:735-49.

3. Borzutzky A, Stern S, Reiff A, Zurakowski D, Steinberg EA, Dedeoglu F, et al. Pediatric chronic nonbacterial osteomyelitis. Pediatrics. 2012;130:e1190-e7.

4. Golla A, Jansson A, Ramser J, Hellebrand H, Zahn R, Meitinger T, et al. Chronic recurrent multifocal osteomyelitis (CRMO): evidence for a susceptibility gene located on chromosome 18q21.3-18q22. Eur J Hum Genet. 2002;10:217-21.

5. Jansson A, Renner ED, Ramser J, Mayer A, Haban M, Meindl A, et al. Classification of non-bacterial osteitis: retrospective study of clinical, immunological and genetic aspects in 89 patients. Rheumatology (Oxford). 2007;46:154-60.
6. Ferguson PJ, El-Shanti HI. Autoinflammatory bone disorders. Curr Opin Rheumatol. 2007;19:492-8.

7. Schnabel A, Range U, Hahn G, Siepmann T, Berner R, Hedrich $\mathrm{CM}$. Unexpectedly high incidences of chronic non-bacterial as compared to bacterial osteomyelitis in children. Rheumatol Int. 2016;36:1737-45.

8. Ferguson PJL, Laxer RM. Autoinflammatory bone disorders. In: Petty L, Lindsley, Wedderburn, editors. Textbook of pediatric rheumatology. 7th ed. Philadelphia: Elsevier; 2016. p. 627-41.

9. Morbach H, Hedrich CM, Beer M, Girschick HJ. Autoinflammatory bone disorders. Clin Immunol. 2013;147:185-96.

10. Sharma M, Ferguson PJ. Autoinflammatory bone disorders: update on immunologic abnormalities and clues about possible triggers. Curr Opin Rheumatol. 2013;25:658-64.

11. Hedrich CM, Hofmann SR, Pablik J, Morbach H, Girschick HJ. Autoinflammatory bone disorders with special focus on chronic recurrent multifocal osteomyelitis (CRMO). Pediatr Rheumatol Online J. 2013;11:47.

12. Girschick HJ, Zimmer C, Klaus G, Darge K, Dick A, Morbach H Chronic recurrent multifocal osteomyelitis: what is it and how should it be treated? Nat Clin Pract Rheumatol. 2007;3:733-8.

13. Hofmann SR, Schnabel A, Rosen-Wolff A, Morbach H, Girschick HJ, Hedrich CM. Chronic nonbacterial osteomyelitis: pathophysiological concepts and current treatment strategies. J Rheumatol. 2016;43:1956-64.

14. Vittecoq O, Said LA, Michot C, Mejjad O, Thomine JM, Mitrofanoff P, et al. Evolution of chronic recurrent multifocal osteitis toward spondylarthropathy over the long term. Arthritis Rheum. 2000;43:109-19.

15. Wipff J, Costantino F, Lemelle I, Pajot C, Duquesne A, Lorrot M, et al. A large national cohort of French patients with chronic recurrent multifocal osteitis. Arthritis Rheumatol. 2015;67:1128-37.

16. Jansson AF, Grote V, ESPED Study Group. Nonbacterial osteitis in children: data of a German Incidence Surveillance Study. Acta Paediatr. 2011;100:1150-7.

17. Jansson AF, Muller TH, Gliera L, Ankerst DP, Wintergerst U, Belohradsky $\mathrm{BH}$, et al. Clinical score for nonbacterial osteitis in children and adults. Arthritis Rheum. 2009;60:1152-9.

18. Probst FP, Bjorksten B, Gustavson KH. Radiological aspect of chronic recurrent multifocal osteomyelitis. Ann Radiol (Paris). 1978;21:115-25.

19. Jurik AG. Chronic recurrent multifocal osteomyelitis. Semin Musculoskelet Radiol. 2004;8:243-53.

20. Duffy CM, Lam PY, Ditchfield M, Allen R, Graham HK. Chronic recurrent multifocal osteomyelitis: review of orthopaedic complications at maturity. J Pediatr Orthop. 2002;22:501-5.

21. Ferguson PJ, Sykelyk A, Figueredo R, Koropatnick J. Synergistic cytotoxicity against human tumor cell lines by oncolytic adenovirus d11520 (ONYX-015) and melphalan. Tumori. 2016;102:31-9.

22. Jurik AG, Egund N. MRI in chronic recurrent multifocal osteomyelitis. Skelet Radiol. 1997;26:230-8.

23. Morbach H, Schneider P, Schwarz T, Hofmann C, Raab P, Neubauer $\mathrm{H}$, et al. Comparison of magnetic resonance imaging and $99 \mathrm{mTechnetium-labelled} \mathrm{methylene} \mathrm{diphosphonate} \mathrm{bone} \mathrm{scin-}$ tigraphy in the initial assessment of chronic non-bacterial osteomyelitis of childhood and adolescents. Clin Exp Rheumatol. 2012;30: 578-82.

24. Hofmann C, Wurm M, Schwarz T, Neubauer H, Beer M, Girschick $\mathrm{H}$, et al. A standardized clinical and radiological follow-up of patients with chronic non-bacterial osteomyelitis treated with pamidronate. Clin Exp Rheumatol. 2014;32:604-9.

25. Khanna G, Sato TS, Ferguson P. Imaging of chronic recurrent multifocal osteomyelitis. Radiographics. 2009;29:1159-77.

26. Ferguson PJ, Chen S, Tayeh MK, Ochoa L, Leal SM, Pelet A, et al. Homozygous mutations in LPIN2 are responsible for the syndrome of chronic recurrent multifocal osteomyelitis and congenital 
dyserythropoietic anaemia (Majeed syndrome). J Med Genet. 2005;42:551-7.

27. Twilt M, Laxer RM. Clinical care of children with sterile bone inflammation. Curr Opin Rheumatol. 2011;23:424-31.

28. Wipff J, Adamsbaum C, Kahan A, Job-Deslandre C. Chronic recurrent multifocal osteomyelitis. Joint Bone Spine. 2011;78:55560.

29. El-Shanti H, Ferguson P. Majeed syndrome. In: Adam MP, Ardinger HH, Pagon RA, Wallace SE, Bean LJH, Mefford HC, Stephens K, Amemiya A, Ledbetter N, editors. GeneReviews®. Seattle: University of Washington; 1993-2017.

30. El-Shanti HI, Ferguson PJ. Chronic recurrent multifocal osteomyelitis: a concise review and genetic update. Clin Orthop Relat Res. 2007;462:11-9.

31. Beck C, Girschick HJ, Morbach H, Schwarz T, Yimam T, Frenkel J, et al. Mutation screening of the IL-1 receptor antagonist gene in chronic non-bacterial osteomyelitis of childhood and adolescence. Clin Exp Rheumatol. 2011;29:1040-3.

32. Aksentijevich I, Masters SL, Ferguson PJ, Dancey P, Frenkel J, van Royen-Kerkhoff A, et al. An autoinflammatory disease with deficiency of the interleukin-1-receptor antagonist. N Engl J Med. 2009;360:2426-37.

33. Ferguson PJ, Laxer RM. New discoveries in CRMO: IL-1beta, the neutrophil, and the microbiome implicated in disease pathogenesis in Pstpip2-deficient mice. Semin Immunopathol. 2015;37:407-12.

34. Valdearcos M, Esquinas E, Meana C, Pena L, Gil-de-Gomez L, Balsinde $\mathrm{J}$, et al. Lipin-2 reduces proinflammatory signaling induced by saturated fatty acids in macrophages. J Biol Chem. 2012;287:10894-904.

35. Herlin T, Fiirgaard B, Bjerre M, Kerndrup G, Hasle H, Bing X, et al. Efficacy of anti-IL-1 treatment in Majeed syndrome. Ann Rheum Dis. 2013;72:410-3

36. Reddy S, Jia S, Geoffrey R, Lorier R, Suchi M, Broeckel U, et al. An autoinflammatory disease due to homozygous deletion of the IL1RN locus. N Engl J Med. 2009;360:2438-44.

37. Omenetti A, Carta S, Caorsi R, Finetti M, Marotto D, Lattanzi B, et al. Disease activity accounts for long-term efficacy of IL-1 blockers in pyogenic sterile arthritis pyoderma gangrenosum and severe acne syndrome. Rheumatology (Oxford). 2016;55:1325-35.

38. Hedrich CM, Bream JH. Cell type-specific regulation of IL-10 expression in inflammation and disease. Immunol Res. 2010;47:185206.

39. McInnes IB. Cytokines. In: Firesetin GS, Budd RC, Sherine EG, McInnes IB, O'Dell SR, editors. Kelley and Firestein's textbook of rheumatology, vol. 10. Philadelphia: Elsevier; 2017. p. 396-407.

40. Hamel J, Paul D, Gahr M, Hedrich CM. Pilot study: possible association of IL10 promoter polymorphisms with CRMO. Rheumatol Int. 2012;32:555-6.

41.• Hofmann SR, Kubasch AS, Ioannidis C, Rosen-Wolff A, Girschick $\mathrm{HJ}$, Morbach H, et al. Altered expression of IL-10 family cytokines in monocytes from CRMO patients result in enhanced IL-1beta expression and release. Clin Immunol. 2015;161:300-7. Report on mechanisms resulting in increased inflammasome activation in human CNO/CRMO.

42. Hofmann SR, Morbach H, Schwarz T, Rosen-Wolff A, Girschick HJ, Hedrich CM. Attenuated TLR4/MAPK signaling in monocytes from patients with CRMO results in impaired IL-10 expression. Clin Immunol. 2012;145:69-76.

43. Hofmann SR, Schwarz T, Moller JC, Morbach H, Schnabel A, Rosen-Wolff A, et al. Chronic non-bacterial osteomyelitis is associated with impaired Sp1 signaling, reduced IL10 promoter phosphorylation, and reduced myeloid IL-10 expression. Clin Immunol. 2011;141:317-27.

44. Greenhill CJ, Jones GW, Nowell MA, Newton Z, Harvey AK, Moideen AN, et al. Interleukin-10 regulates the inflammasomedriven augmentation of inflammatory arthritis and joint destruction.
Arthritis Res Ther. 2014;16:419. Report on effects of increased inflammasome activation on bone loss in mice.

45. Scianaro R, Insalaco A, Bracci Laudiero L, De Vito R, Pezzullo M, Teti A, et al. Deregulation of the IL-1beta axis in chronic recurrent multifocal osteomyelitis. Pediatr Rheumatol Online J. 2014;12:30.

46. Nakashima T, Takayanagi H. Osteoimmunology: crosstalk between the immune and bone systems. J Clin Immunol. 2009;29:555-67.

47. Nakashima T, Takayanagi H. Osteoclasts and the immune system. J Bone Miner Metab. 2009;27:519-29.

48. Hofmann SR, Rosen-Wolff A, Tsokos GC, Hedrich CM. Biological properties and regulation of IL-10 related cytokines and their contribution to autoimmune disease and tissue injury. Clin Immunol. 2012;143:116-27.

49. Cox A, Darbro B, Laxer R, Bing X, Finer A, Erives A, et al. Recessive coding and regulatory mutations in FBLIM1 underlie the pathogenesis of sterile osteomyelitis [abstract]. Arthritis Rheumatol. 2016;68(suppl 10). http://acrabstracts.org/abstract/ recessive-coding-and-regulatory-mutations-in-fblim1-underlie-thepathogenesis-of-sterile-osteomyelitis/.

50.• Cox AJ, Darbro BW, Laxer RM, Velez G, Bing X, Finer AL, et al. Recessive coding and regulatory mutations in FBLIM1 underlie the pathogenesis of chronic recurrent multifocal osteomyelitis (CRMO). PLoS One. 2017; in press. Report on genetic causes of human CNO/CRMO.

51. Hofmann SR, Kubasch AS, Range U, Laass MW, Morbach H, Girschick HJ, et al. Serum biomarkers for the diagnosis and monitoring of chronic recurrent multifocal osteomyelitis (CRMO). Rheumatol Int. 2016;36:769-79.

52. Chitu V, Nacu V, Charles JF, Henne WM, McMahon HT, Nandi S, et al. PSTPIP2 deficiency in mice causes osteopenia and increased differentiation of multipotent myeloid precursors into osteoclasts. Blood. 2012;120:3126-35.

53. Grosse J, Chitu V, Marquardt A, Hanke P, Schmittwolf C, Zeitlmann L, et al. Mutation of mouse Mayp/Pstpip2 causes a macrophage autoinflammatory disease. Blood. 2006;107:3350-8.

54. Ferguson PJ, Bing X, Vasef MA, Ochoa LA, Mahgoub A, Waldschmidt TJ, et al. A missense mutation in pstpip2 is associated with the murine autoinflammatory disorder chronic multifocal osteomyelitis. Bone. 2006;38:41-7.

55. Byrd L, Grossmann M, Potter M, Shen-Ong GL. Chronic multifocal osteomyelitis, a new recessive mutation on chromosome 18 of the mouse. Genomics. 1991;11:794-8

56. Hentunen TA, Choi SJ, Boyce BF, Dallas MR, Dallas SL, ShenOng GL, et al. A murine model of inflammatory bone disease. Bone. 2000;26:183-8.

57. Liao HJ, Chyuan IT, Wu CS, Lin SW, Chen KH, Tsai HF, et al. Increased neutrophil infiltration, IL-1 production and a SAPHO syndrome-like phenotype in PSTPIP2-deficient mice. Rheumatology (Oxford). 2015;54:1317-26.

58. Ahmed S, Bu W, Lee RT, Maurer-Stroh S, Goh WI. F-BAR domain proteins: families and function. Commun Integr Biol. 2010;3:116 21.

59. Tsujita K, Kondo A, Kurisu S, Hasegawa J, Itoh T, Takenawa T. Antagonistic regulation of F-BAR protein assemblies controls actin polymerization during podosome formation. J Cell Sci. 2013;126: 2267-78.

60. Cassel SL, Janczy JR, Bing X, Wilson SP, Olivier AK, Otero JE, et al. Inflammasome-independent IL-1beta mediates autoinflammatory disease in Pstpip2-deficient mice. Proc Natl Acad Sci U S A. 2014;111:1072-7.

61.• Lukens JR, Gross JM, Calabrese C, Iwakura Y, Lamkanfi M, Vogel $\mathrm{P}$, et al. Critical role for inflammasome-independent IL-1beta production in osteomyelitis. Proc Natl Acad Sci U S A. 2014;111: 1066-71. Report on mechanisms resulting in increased IL-1 expression in murine CNO/CRMO. 
62.• Netea MG, van de Veerdonk FL, van der Meer JW, Dinarello CA, Joosten LA. Inflammasome-independent regulation of IL-1-family cytokines. Annu Rev Immunol. 2015;33:49-77. Report on mechanisms resulting in increased IL-1 expression in murine $\mathrm{CNO}$ / CRMO.

63. Lukens JR, Gurung P, Vogel P, Johnson GR, Carter RA, McGoldrick DJ, et al. Dietary modulation of the microbiome affects autoinflammatory disease. Nature. 2014;516:246-9. Report on potential effects of the microbiome in murine CNO/CRMO.

64. Ter Horst R, Jaeger M, Smeekens SP, Oosting M, Swertz $\mathrm{MA}, \mathrm{Li} \mathrm{Y}$, et al. Host and environmental factors influencing individual human cytokine responses. Cell. 2016;167:111124. e13

65. Schnabel A, Range U, Hahn G, Berner R, Hedrich CM. Treatment response and longterm outcomes in children with chronic nonbacterial osteomyelitis. J Rheumatol. 2017;44(7):1058-65. Reports on long-term outcomes in CNO/CRMO.

66. Beck C, Morbach H, Beer M, Stenzel M, Tappe D, Gattenlohner S, et al. Chronic nonbacterial osteomyelitis in childhood: prospective follow-up during the first year of anti-inflammatory treatment. Arthritis Res Ther. 2010;12:R74. 